\title{
Monitoring of Honey Bee Hiving System using Sensor Networks
}

\author{
Sachin K ${ }^{1}$, Gagana M. R ${ }^{2}$, Heema Rubab ${ }^{3}$, Jalaja G S ${ }^{4}$, Jayanand J 5 \\ Department of Electronics \& Communication Engineering \\ ${ }^{2-5}$ Eight Semester UG Students, AIET, Moodbidri, Karnataka, India. \\ ${ }^{1}$ Assistant Professor, AIET, Moodbidri, Karnataka, India.
}

\begin{abstract}
Honey bees have throughout history been a keystone species, pollinating an estimated 70 percent of all plants and underpinning some 30 percent of the global food supply. Because the viability of beehives is a critical predictor of the planet's future health and agricultural sustainability, reports of a precipitous decline in the number of colonies around the world have stirred considerable alarm. Since most of the prior contributions are focusing on data gathering, the approach to focus on the user's needs is central to take next steps in the field of using sensors for Beekeeping. Beekeepers can be divided into beekeepers having bees as a hobbies and beekeepers that are professional, making a living of the beekeeping. Visualization and availability of data are key questions for user friendliness. Since there are no standards for measurement data from beehives, there are different manufacturers/contributors that have their own system. If a standard format would be available, it would make it easier to interconnect different devices for visualization in single user interface. If data is available as streams in standardized application program interface (API) a user can use whatever solution found for visualization. The majorities of beekeepers that non-professional, they will probably want to have the "relation to their bees", using too much technology is probably wanted, like having robots doing the actual beekeeping work.
\end{abstract}

Keywords- Application Program Interface (API).

\section{INTRODUCTION}

Honey bees are important for pollination of crops. Honeybees produce different functionalities in our ecosystem, where the two main outcomes are the pollination of crops and honey. There are other products from the bees including Royal Jelly, Pollen and Propolis that are produced by bees. Bees are important for the global food supply, as the quantity and quality of food are increased by pollination, and honeybees are a major pollinator. Crops, including fruits like strawberries are dependent on pollination from bees. The products that are produced by bees need to be harvested and handled from the beehives, normally done by a beekeeper. The beekeeper either keeps bees as a hobby or as a profession where the outcome is important.

Since many years humans have tried to harvest honey from the bees, a proof of activities are old cave paintings approximately 8000-9000 years old. The other technical improvement is the queen excluder, which makes a "barrier" between the area where the queen lay eggs, and the other side of the barrier where only worker bees smaller can pass. The queen excluder makes boxes with only honey, and no eggs, easier to handle for the beekeeper. These two improvements made beekeeping easier and more efficient to product. The bees are normally kept in hives, consisting of "houses". Inside the hive there are frames with cells of wax. The wax is a product of the bees. In a cell the bees can store pollen, honey or have a new bee produced. The bees are produced by the queen, which lay eggs in cells, and then the worker bees are feeding the egg to become a bee in 21 days.

The high proportion of non-professional beekeepers and the number of colonies per beekeeper were the only common characteristics at European level. This fact need to be taken into consideration, that the main group when it comes to beekeepers are not mandatory to make a living from their beekeeping. Today beehives are placed not only on the traditional countryside, but also in the cities. The reason could probably be that people are becoming more aware of the positive side of honeybees.

A main part of beekeeping is the manual inspection of beehives, a "craftsmanship" where the person doing the activities must understand the nature, what to observe and what to actions to take. Reading books, papers can helpful, especially for understanding, but when it comes to observation, judgments, comparison, and actions, experience will help the beekeeper to do a better job. The manual inspection of a beehive is assumed to be done by a beekeeper or a group of beekeepers wearing protective clothes. The aim of a visit to the beehive, are observation, probably some decision-making from observations, and some actions. Depending on the time of year, there are different activities in the beehive, also the local weather conditions affect the activities. Beekeepers are normally doing manual inspections of their hives, to check weight, health etc. To observe the bee health, the reproduction is always interesting. So, checking for eggs, pupae and cells of bees are central when it comes to observation. The number of eggs/larvae/pupae need to be put in context of the time of year, the natures current situation and the specific beehives specific situation. If there are eggs, a queen is present, if the pupae are "normal" it's a mated queen laying eggs. If you don't have fresh eggs during active season, there are probably a problem, then you need to locate a queen visually, and probably take some action to provide a new queen. Another thing to observe is, if there are pollen and nectar present in the nature, one could expect a lively colony, with many bees, much honey, some of it already. The weight of the hive is interesting during the whole year, are there enough food during low season, and how 
much honey can I expect? During winter time, a beekeeper can feel the heat under the top of the roof separated by plastic to avoid stinging, which indicates a healthy society.

\section{MOTIVATION}

- The motivation for doing this project is to design an automation system for apiculture field.

- This project proposes a new simpler framework for the raw data such as brood temperature, brood humidity, ambient temperature and weight of the hive can also be accessed from the cloud server by the beekeepers.

\section{PROBLEM STATEMENT}

- Farmer has to go near hiving to check their productivity like weight of honey, temperature and humidity etc.

- Whenever apiculturist is going near to the honey hiving, there is $3 \%$ of the honey bee will be lost.

- If this process happens daily there will be loss in productivity.

- Repetitive tasks are not comfort with manpower as shown in Fig 1.5.

- During a winter season there will be destruction of honeycomb structure due to formation of hosts on honeycomb hive in turn there will be loss in productivity as shown in Fig 1.4.

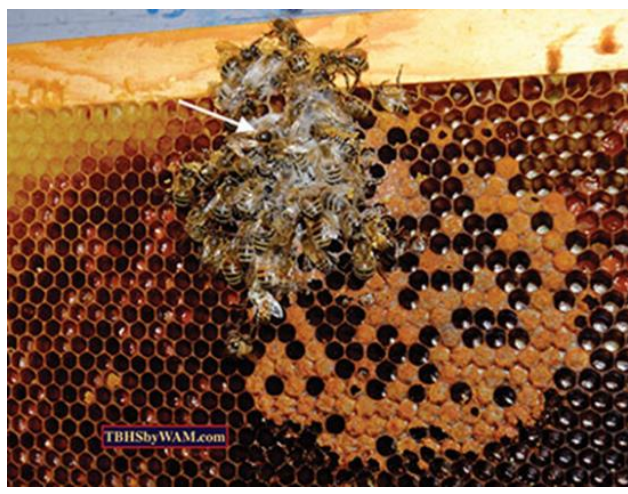

Fig.1.4 Loss in production

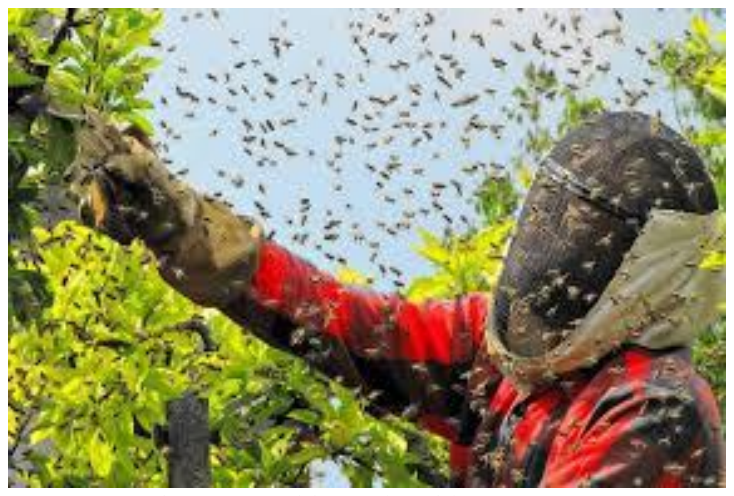

Fig.1.5 Repetitive tasks with manpower

\section{OBJECTIVES}

- To know the weight of the honey and free from human intervention.

- To know the temperature inside the honey comb.

- To know the humidity inside the honey comb.

- To check whether the Queen bee is present or

\section{II.LITERATURE REVIEW}

Anand et al [1] Honey bee holding is a deep rooted practice of holding colony states for acquiring nectar and other apiary based absolutely items and is in fact named as apiculture. This paper proposed a gadget that identifies swarming the utilization of two remarkable methodologies. The first being, utilizing sound-related assessment of the sound created by utilizing the honey bees all through swarming. What's more, the subsequent methodology, recognition by methods for following microclimatic changes and varieties inside the weight. Once swarming is detected the gadget is designed to alert the apiculturist by cloud services. The measurements together with brood temperature, brood humidity, surrounding temperature and weight of the hive can likewise be gotten to from the cloud server with the guide of the honey bee attendants, subsequently making the colony following procedure free from human intercession.

Daniel M. Lofaro et al [2] suggested that honey bees are a significant piece of our reverberation framework. Pollinators, comprising of honey bees, help in any event $30 \%$ of the world's yields and $90 \%$ of the world's wild intends to flourish through cross-fertilization. Studies show that among 2015 and 2016 beekeepers lost $44 \%$ of honey bee settlements over the winter that is up around $10 \%$ from the previous decade. Foundations for this decrease is associated to the general hive wellbeing. The more sound the hive the more noteworthy likely it's far to keep on existing the winter. Current time directs people check the hives for Varro Mite pervasions, awful dampness levels, and diverse telling side effects of an undesirable hive. These checks are obtrusive to the honey bee provinces and just happen a few times each year. Foundations for this decrease is associated to the general hive wellbeing. The more sound the hive the more noteworthy likely it's far to keep on existing the winter.

Zacepins et al [3] recommended that honey bee wintering process is one of the natural procedures, which these days might be checked and controlled the utilization of the data innovation, control strategies and devices. Control and following of such method is truly entangled and no longer an insignificant errand, since direct of the natural gadget, natural items and their reaction to human mediation is erratic. To entire this undertaking it's far imperative to assemble complex control machine engineering with numerous subsystems and components. The automatized framework engineering proposed through the creators is created to upgrade the honey bee wintering process and to consolidate realities advancements into this technique. The machine is uncommonly utilized for singular bee state checking and control.

Edwards Murphy et al [4] Checking the sounds discharged with the assistance of honey bees for the term of an apiary is a basic side interest for beekeepers to frame positive that their honey bee provinces stay solid and to augment productiveness. 
A one of a kind conceptive event called "swarming", incorporates the flight of a sovereign with an outsized a piece of the hive masses. Swarming are frequently recognized through sound, and whenever permitted to happen unchecked may result during a drop by means of hive efficiency or, in extreme cases, settlement demise. A method for effectively alarming the manager that such an occasion is inevitable would asset in protective the bumble bee populace and diminishing beekeeping costs. During this paper, an answer the utilization of off the rack Wireless Sensor Network (WSN) time with low power sign handling to show settlement sound, likewise because of the reality the temperature and mugginess of the hive inside is introduced.

Rodriguez et al [5] proposed an acknowledgment framework for dust bearing honey bees from motion pictures of the passageway of the hive is introduced. This $\mathrm{pc}$ vision undertaking can be a key part for the mechanized observing of honey bees with an end goal to get large scope data of their scavenging behavior and task specialization. A few methods are contemplated for this task, alongside gauge classifiers, shallow Convolution Neural Networks, and more profound systems from the writing. The trial differentiate depends on a substitute dataset of depictions of bumble bees that was physically explained for the nearness of dust. The proposed technique, upheld Convolution Neural Networks is appeared to beat the contrary procedures in expressions of exactness. Detailed examination of the outcomes and accordingly the influence of the building parameters, similar with the impact of devoted shading based data increase, offer bits of knowledge into the best approach to apply the method to the objective application.

\section{METHODOLOGY}

The NodeMCU based on ESP8266 was used as the wireless mesh node. BLYNK server is an open source Internet of things (IOT) cloud server. The microclimatic parameters within the hive are measured by DHT11 sensor, whereas the temperature right outside the hive is measured by BMP180.The system is proposed to design the monitoring of parameters that indicate swarming and alert the beekeeper at the instant pre-swarming is initiated, through the cloud servers in an efficient and effective manner.

Typical block diagram as shown in Fig 1.1 consists of NodeMCU which is connected to DHT11, BMP180 power supply and load cell. The design of the proposed system revolves around maximum functionality in the practical environment and at the same time is developed to be user friendly, compact and completely built on the Microcontroller platform.

The system proposed in this project is used to monitor the parameters that indicate swarming and alert the beekeeper at the instant pre-swarming is initiated, through the cloud servers in an efficient and effective manner. To ensure that bees don't leave the apiary during proliferation it is necessary to detect swarming as soon as it is initiated.

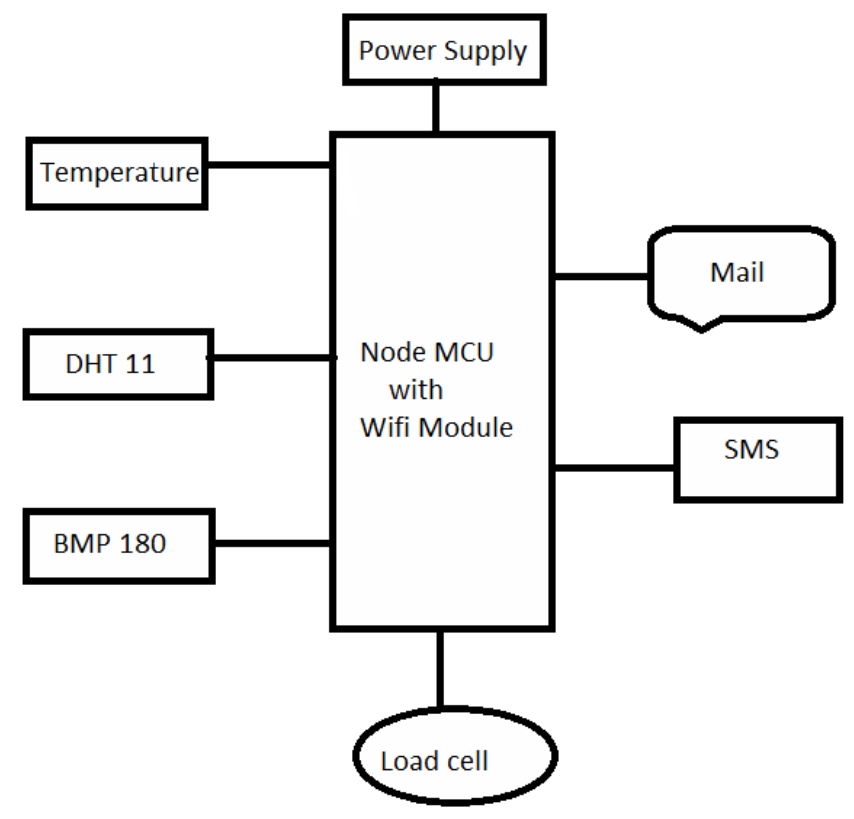

Fig.1.1 Block diagram of proposed system

- $\quad$ NodeMCU with the ESP8266. BMP180, DHT11 and the load cell are the sensors used in the system to monitor temperature, humidity.

- An electrolyte is used to pick up the sound produced by the bees that is used for auditory analysis.

- The NodeMCU based on ESP8266 was used as the wireless mesh node.

- The cloud server of choice is BLYNK an open source IOT cloud server.

- This is implemented on star network topology and hence made bridging between the mesh and a star network which is complex and non-trivial task.

- The NodeMCU RF transceiver supports the IEEE $802.11 \mathrm{~b} / \mathrm{g} / \mathrm{n}$ standards, with which the mesh network is implemented.

- $\quad$ The distance between each node can go up 90 meters with a line of sight. When not in line of sight their range was measured up to 20 meters.

\section{RESULTS}

The project proposed a low-cost, reliable beehive-monitoring system based on a WSN to measure the temperature, relative humidity, and weight of beehives in real time and nonintrusively. Unlike other beehive-monitoring systems, Bee performs the synchronized acquisition of samples from all hives of an apiary. This aspect is fundamental for future analysis of the data and their comparison between different hives.

\section{APPLICATIONS}

The applications of the proposed project are:

- The smart hiving technology allows beekeepers to receive all information related to bees inside the hive at regular intervals of time.

- It will be made possible to hive under unfavorable weather conditions. 
- By using the sensor network whole farm can be brought into control.

\section{ADVANTAGES}

Autonomous design reduces human errors in controlling, thus reducing labor dependency.

- Reduces the labor charges.

- Having temperature and pressure sensors helps to get the perfect yield.

\section{CONCLUSION}

This paper which mainly deals beekeepers in general, are nature interested people and can see their bees as pets. Listening to bees on remote would probably be nice, having automatic analysis of the sound would make a detection system, as the probably will do. Using weather data in combination with weight could serve as a good detection system for bees during winter/spring for "need to feed", and during summer season, "time for expansion" or "time to harvest". However, all this data needs to be analyzed in the context of beekeeping. All beekeepers have different routines and details in their methods. Andersson means a beekeeper have no use of information, if there are no plans for actions, due to the information. Since there are majorities of beekeepers that non-professional, they will probably want to have the "relation to their bees", using too much technology is probably not wanted, like having robots doing the actual beekeeping work.

\section{VIII.ACKNOWLEDGEMENT}

It is my proud privilege and duty to acknowledge the kind of help and guidance received from several people in preparation of this report. It would not have been possible to prepare this report in this form without their valuable help, cooperation and guidance.

First and foremost, I wish to record my sincere gratitude to the Management and to our beloved Principal, Dr. Peter Fernandes, Alva's Institute of Engineering and Technology, Moodbidri for his constant support and encouragement and for making available library and internet facilities needed to prepare this report.

I would like to thank Dr. D.V. Manjunatha, Head, Department of Electronics and Communication Engineering, AIET, for his valuable suggestions and guidance throughout the period of this report.

I would like to express my sincere gratitude to my guide, Mr Sachin K, Assistant Professor, Department of Electronics and Communication Engineering, AIET, Moodbidri for his guidance, encouragement and inspiration.

I am thankful to my Project Coordinator, $\mathbf{M r}$ Santosh, Assistant Professor, Department of Electronics and Communication Engineering, AIET, Moodbidri for her valuable guidance, encouragement and co-operation during the course of the seminar and presentation.

\section{REFERENCES}

- Anand [1], the work presented in this paper was focused on developing a system that could help monitor the beehive and detect swarming by two methods.

- $\quad$ Daniel M. Lofaro [2], proposed that bees are an important part of our echo system. Pollinators, such as bees, help at least $30 \%$ of the world's crops and $90 \%$ of the world's wild plans to thrive via crosspollination.

- Zacepins [3], proposed that Honey bee wintering process is one of the biological processes, which nowadays can be monitored and controlled using the information technologies, control methods and tools.

- $\quad$ Edwards Murphy [4], Monitoring the sounds emitted by honey bees in a beehive is an important activity for beekeepers to ensure that their bee colonies remain healthy and to maximise productivity.

- $\quad$ Rodriguez [5], proposed a recognition system for pollen bearing honey bees from videos of the entrance of the hive is presented.

- Zacepins [6], proposed that implementation of precision apiculture methods into practice is highly depended on availability and costeffectiveness of honey bee monitoring equipment.

- $\quad$ Edwards Murphy [7], proposed that honey bees have held a critical role in agriculture and nutrition from the dawn of human civilization.

- Thomas D. Seeley [8], proposed a paper that says Worker piping, which is reported only in hives, was observed in swarms as they prepared to lift-off to fly to a new home. 